\title{
Impact of Surgical Techniques in Maternal Morbidity and Foetal Outcome in Obstructed Labour: A Retrospective Study of 100 Cases of Obstructed Labour.
}

\author{
Anujeet Kaur Randhawa ${ }^{1,}$ Dr.Amarbir Singh ${ }^{2}$, Dr. Sudershan Kapoor ${ }^{3}$, \\ ${ }^{1}$ Senior Resident, Department Of Obstetrics And Gynaecology, Bebe Nanki Mother And Child Care Center, \\ Government Medical College, Amritsar, Punjab, India. \\ ${ }^{2}$ Asst. Prof. Department Of Surgery, Govt. Medical College, Amritsar, Punjab, India. \\ ${ }^{3}$ Prof. Department Of Surgery, Govt. Medical College, Amritsar, Punjab, India.
}

\begin{abstract}
:
Background: To compare the maternal and neonatal morbidities between the "Patwardhan" technique, reverse breech and the vertex extraction (Push method) of the foetus in second stage caesarean sections.

Methods: A Retrospective study was done at Department of Obstetrics and Gynaecology, Bebe Nanki Mother and Child Care Center, Government College, Amritsar, Punjab, India from year 2013 to 2016 (three and half years). Women with full term pregnancy with fetus in vertex position and the head deeply impacted in pelvis and needing caesarean delivery were included in the study.

Group-A consists of all cases in which extraction of foetus was done by Patwardhan technique as well as reverse breech extraction.

Group-B consists of cases in whom extraction of foetus was done by push up method and extracted as vertex.

Objective of the study was to assess selective complications like extension of the incision, injury to the surrounding structures, excessive bleeding, need for blood transfusion and the foetal outcome between the two groups.

Results:- Out of 100 cases reviewed, 55 belonged to group A (Patwardhan and reverse breech extraction) and 45 belonged to group $B$ (vertex extraction). Patients in the group B had statistically significant higher rates of maternal morbidity in terms of uterine extension and other related complications. However; there were no differences in neonatal outcomes in both the groups.

Conclusions: While complications are inherent in methods, Patwardhan method and reverse breech extraction of delivery of the foetus for second stage labour has been shown to confer considerable advantage in prevention of maternal morbidity over the push method in our institution. Our findings support the fact that the Patwardhan method and reverse breech extraction could be a useful manoeuvre in intraoperative disengagement of foetal head, when encountered at second stage CS and it is our opinion that these techniques can be practiced selectively as a primary technique.
\end{abstract}

Keywords: Second stage caesarean section, deeply engaged foetal head, Patwardhan technique, reverse breech extraction, Push method etc.

\section{Introduction}

The desire of every woman in Labour and her obstetrician is to have a normal vaginal birth. During this due course, unanticipated cephalopelvic disproportion at times may end up in deeply impacted head with features of obstructed labour and there is no way other than to deliver these babies by abdominal route. The most frequent cause of obstructed labour is cephalo-pelvic disproportion - a mismatch between the foetal head and the mother's pelvic brim. The foetus may be large in relation to the maternal pelvic brim, such as the foetus of a diabetic woman, or the pelvis may be contracted, which is more common when malnutrition is prevalent. Some other causes of obstructed labour may be malpresentation or malposition of the foetus (shoulder, brow or occipito-posterior positions). In rare cases, locked twins or pelvic tumours can cause obstruction (1). Neglected obstructed labour (OL) is a major cause of both maternal and newborn morbidity and mortality. The obstruction can only be alleviated by means of an operative delivery, either caesarean section or other instrumental delivery (forceps, vacuum extraction or symphysiotomy). Maternal complications include intrauterine infections following prolonged rupture of membranes, trauma to the bladder and/or rectum due to pressure from the foetal head or damage during delivery, and ruptured uterus with consequent haemorrhage, shock or even death. Trauma to the bladder during vaginal or instrumental delivery may lead to stress incontinence. By far the most severe and distressing long-term condition following obstructed labour is obstetric fistula - a hole which forms in the vaginal wall communicating into the bladder (vesico-vaginal fistula) or the rectum (recto-vaginal fistula) or both $(1,2)$. In developing countries, fistulae are commonly the result of prolonged obstructed labour and 
follow pressure necrosis caused by impaction of the presenting part during difficult labour. In the infant, neglected obstructed labour may cause asphyxia leading to stillbirth, brain damage or neonatal death1. Obstructed labour ranked 41st in GBD 1990, representing $0.5 \%$ of the burden of all conditions and $22 \%$ of all maternal conditions.(1,3) it was estimated to be the most disabling of all maternal conditions. According to studies approximately up to $25 \%$ of emergency caesarean sections performed are second stage caesarean sections. The number of second stage caesareans encountered in developing countries is much higher especially in rural population due to neglected obstetric care, poor utilization of available health services , traditional beliefs and practices like preference of home delivery from traditional birth attendants, poor transport facilities, late referrals from primary health care centres where overenthusiastic attempts are made to deliver vaginally in suboptimal condition and also lack of training and confidence to present generation obstetricians to perform instrumental delivery. Caesarean sections done at full cervical dilatation with impacted foetal heads are not only technically difficult, but methods employed for disimpacting the fetal head from the pelvis at caesarean section may perhaps contribute to varying complications. Potential problems include difficulty in delivery of the fetal head, extension of the uterine wound, uterine artery laceration, broad ligament hematoma and a higher risk of postpartum haemorrhage requiring blood transfusion. Fetal complications include injuries, poor APGAR scores and admission to the neonatal ICU unit. Second stage caesareans are always done in emergency and there are a number of widely accepted techniques that obstetrician perform when faced with this potentially difficult task.(5,6) The method chosen may depend upon the skill and experience with a particular method. There is insufficient evidence available to support the use of anyone method. $(8,9)$

Extraction of the impacted fetal head may be done by

1. Push method i.e., pushing through the vagina.(10)

2. Pull method, i.e., a reverse breech technique. $(13,14)$

3. Patwardhan technique i.e. shoulders first technique. (11)

4. Using simple device- Fetal Disimpacting System. (12)

5. Extraction of foetal head with Murless head extractor/ C-snorkel.(12)

One option to deliver impacted fetal head in emergency CS is the conventional head push technique (push method) using an assistant hand in the vagina to push the head up toward the uterine incision while the operator tries to pass his/her hand below the head to dislodge the head from pelvis.(12)

Alternatively a reverse breech extraction technique (pull method) is usually performed by opening the uterus soon to reach into the upper segment for a fetal leg, and by applying gentle traction on the leg until the another leg appeared. Then both legs are held together and the body of fetus could be delivered (pulled) out of the uterus completely using technique similar to that for a breech delivery.$(13,14)$

In Patwardhan's method of delivery in obstructed labour, the incision over lower uterine segment is made at the level of the anterior shoulder of the baby as the head is deeply impacted. Anterior shoulder is then delivered along with the anterior arm by hooking a finger in the elbow if required posterior shoulder is rotated forward and is similarly delivered. The trunk, breech and the lower limbs are successively delivered by traction on arms aided by fundal procedure. Patwardhan Technique. [11] In case of occipito-transverse or occipitoanterior positions with the head deeply impacted in the pelvis, incision is made in the lower uterine segment, at the level of the anterior shoulder, which is delivered out. 2. With gentle traction on this shoulder, the posterior shoulder is also delivered out. 3. Next, the surgeon hooks the fingers through both the axillae and with gentle traction, aided by fundal pressure applied by assistant, the body of the foetus is brought out of the uterus. 4 . Now the baby's head which is the only part of the foetus which is still inside the uterus, is gently lifted out of the pelvis. $(15,16)$

\section{Observations}

In this study of three and half years from 2013 to june 2016, of all the patients who had underwent caesarean section, 100 cases of pregnant women with obstructed labour were taken into consideration for study and observed various parameters:-

\section{Mode of admission}

Almost all the cases of obstructed labour came in emergency (96 out of 100) and 4 patients admitted through O.P.D and get obstructed. 


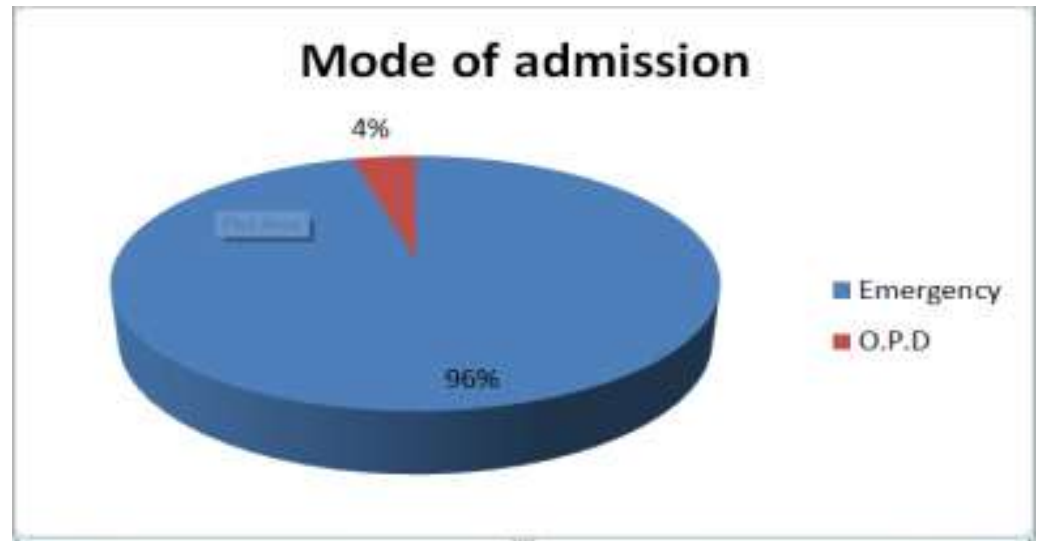

\section{Gravidity}

Out of 100 women with obstructed labour, $75 \%$ of the women were primigravidae, $17 \%$ were second gravidae, $5 \%$ were 3 rd gravidae and $3 \%$ were grand multigravidae.

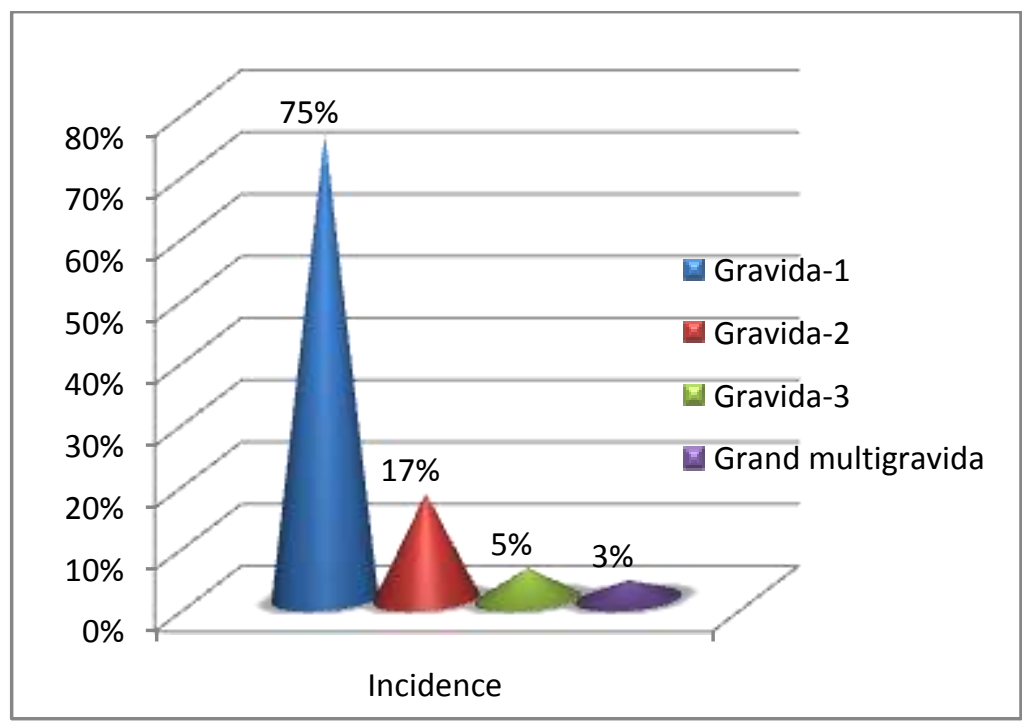

II. Methods of extraction of impacted foetal head in obstructed labour

Out of 100 women with obstructed labour who underwent caesarean section, the various methods were implied to extract foetus. In 45 out of 100 cases foetus were extracted by push up method in vertex position, 33 out of 100 cases Patwardhan technique was implied and in 22 out of 100 cases reverse breech technique was implied.

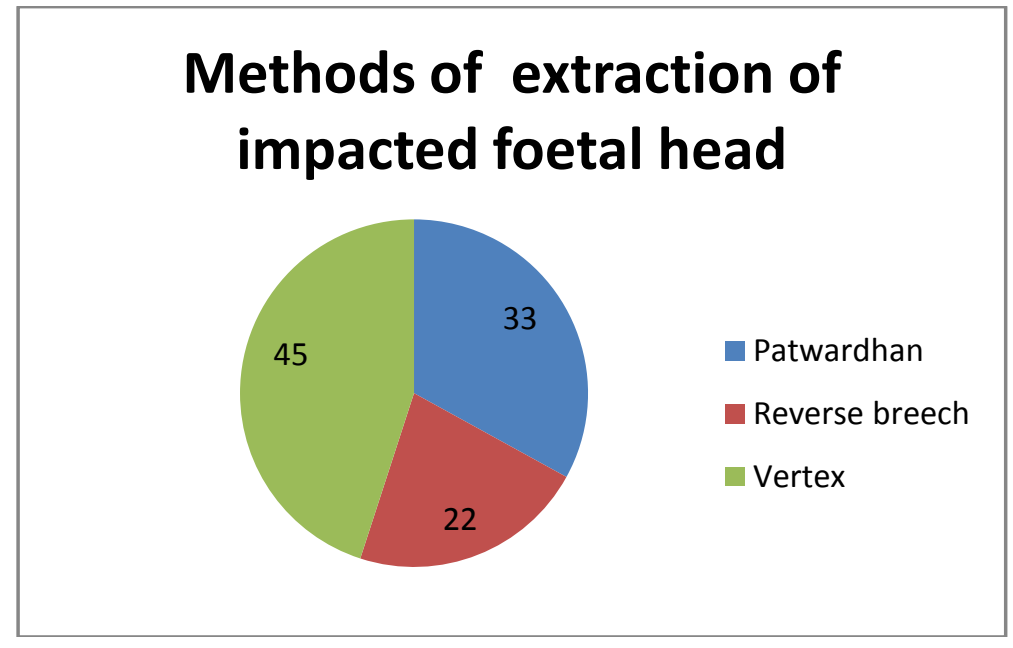




\section{Maternal morbidity}

Various parameters of maternal morbidity such as extension Of uterine Incision, broad ligament hematoma, traumatic PPH, atonic PPH and blood transfusion needed. Extension oF uterine incision was observed in 3(5.45\%) patients in Group- A and 12(26.66\%) patients in Group- B, broad ligament hematoma is observed in $4(8.88 \%)$ patients in Group-B and no case of broad ligament hematoma was encountered in GroupA. Traumatic PPH was observed in 2(3.63\%) patients in Group-A and 9(20\%) patients in Group-B. Atonic PPH was observed in 1 case in Group-A and 3(6.66\%) patients in Group-B and blood transfusion was needed in $12(21.8 \%)$ patients in Grouo-A and 21(46.66\%) patients required blood transfusion in Group-B

\begin{tabular}{|l|l|l|l|}
\hline S.N & Complications & Group - A (55) & Group - B (45) \\
\hline 1. & Extension Of Uterine Incision & $3(5.45 \%)$ & $12(26.66 \%)$ \\
\hline 2. & Broad ligament hematoma & Nil & $4(8.88 \%)$ \\
\hline 3. & Traumatic PPH & $2(3.63 \%)$ & $9(20 \%)$ \\
\hline 4. & Atonic PPH & $1(1.81 \%)$ & $3(6.66 \%)$ \\
\hline 5. & Blood Transfusion Needed & $12(21.8 \%)$ & $21(46.66 \%)$ \\
\hline
\end{tabular}

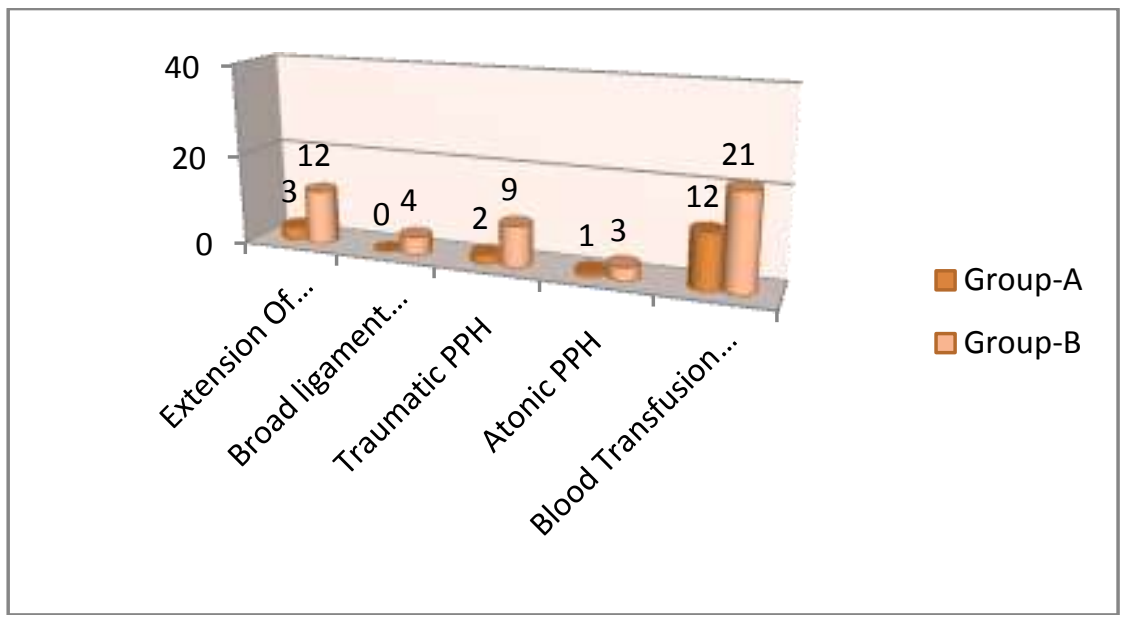

\section{Foetal morbidity and Mortality}

Foetal distress was observed in 10 out of 100 cases out of which 4(7.27\%) cases observed in Group-A and 6(13.33\%) cases in Group-B. Meconium stained liquor was observed in 10(18.18\%) cases in Group-A and $12(26.6 \%)$ cases with meconium stained liquor was observed in Group-B. Still births were 4(7.27\%) cases observed in Group-A and 5(11.11\%) cases in Group-B.

\begin{tabular}{|l|l|l|l|}
\hline S.N & Complications & Group $-\mathrm{A}(55)$ & Group - B(45) \\
\hline 1. & Foetal distress & $4(7.27 \%)$ & $6(13.33 \%)$ \\
\hline 2. & Meconium stained & $10(18.18 \%)$ & $12(26.6 \%)$ \\
\hline 3. & Still births & $4(7.27 \%)$ & $5(11.11 \%)$ \\
\hline
\end{tabular}

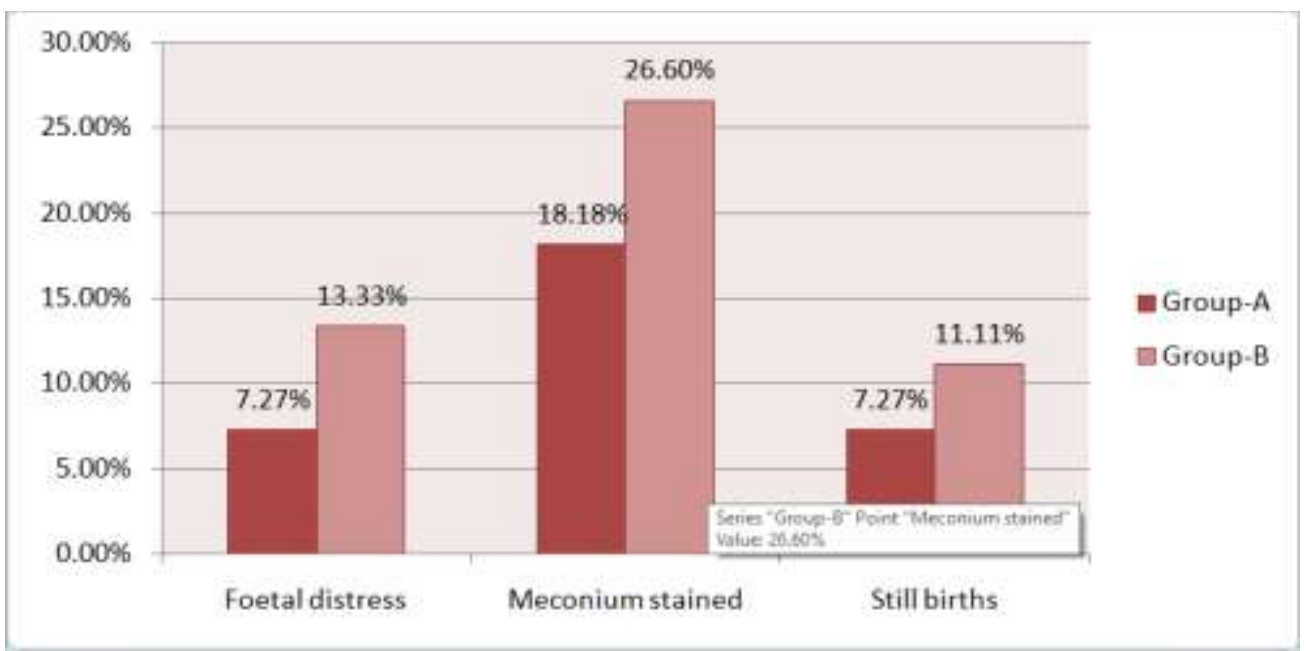




\section{Discussion}

Various parameters of maternal morbidity such as extension Of uterine incision, broad ligament hematoma, traumatic PPH, atonic PPH and blood transfusion needed. Uterine laceration and its related complications increase morbidity and also has a long-term implication on the patient's future obstetric careers as it is a contraindication for allowing subsequent vaginal delivery $(17,18)$. The incidence of extension of incision in second stage caesarean sections seen in push method extraction was found to be about $15 \%$ to $50 \%$ in various studies 13.In our study, It is observed that extension of uterine incision is observed in $3(5.45 \%)$ patients in Group- A and 12(26.66\%) patients in Group- B. The possible explanations for increased incidence of uterine incision extension in push method are 1) Iatrogenic trauma caused by the operating surgeon in manoeuvre his hand between the already stretched, congested lower segment and the impacted head. 2) Push from below, if applied posterior to the flexion point can lead to iatrogenic extension of head in utero, with either face/ brow presentation. 3) Undue force exerted by the operating surgeon in order to lift the deeply impacted head out of pelvis up to a highly placed uterine incision. 4) The tonic contracted uterus on the fetal spine acts as a splint, there by resisting both flexion at atlanto-occipital joint and upward lifting of presenting part. These compel the operating surgeon as well as the assistant pushing from below to apply excessive force, thereby further increasing the chances of uterine extension. By contrast, in Patwardhan method the extraction is partially reverse with shoulder first followed by trunk and breech successively, and head virtually pops out at last. The operating surgeon neither inserts his hand into the cavity nor does he apply any undue force. Hence the chances of maternal injury are lessened by this method. Similarly, in reverse breech method, the extraction of the baby is done by pulling at the foetal leg present in the upper segment and the head comes out at the end.In this method also, no force is directly applied on to the thin oedometous lower segment, thereby safeguarding against maternal injuries. Broad ligament hematoma is observed in $4(8.88 \%)$ patients in Group-B and no case of Broad ligament hematoma was encountered in Group-A. Traumatic PPH was observed in 2(3.63\%) patients in GroupA and 9(20\%) patients in Group-B. Atonic PPH was observed in1 case in Group-A and 3(6.66\%)patients in Group-B and Blood Transfusion was needed in 12(21.8\%) patients in Grouo-A and 21(46.66\%) patients required blood transfusion in Group-B The incidence of incision extension was approximately 5 times more common in push method than Patwardhan method and reverse breech extraction. Uterine incision extension related complications like uterine artery laceration, broad ligament hematoma, bladder injury, traumatic PPH and blood transfusion were all more in push group than in Patwardhan group. Broad ligament hematoma is observed in $4(8.88 \%)$ patients in Group-B and no case of Broad ligament hematoma was encountered in GroupA. Traumatic PPH was observed in 2(3.63\%) patients in Group-A and 9(20\%) patients in Group-B. Atonic PPH was observed in1 case in Group-A and 3(6.66\%)patients in Group-B and Blood Transfusion was needed in $12(21.8 \%)$ patients in Grouo-A and 21(46.66\%) patients required blood transfusion in Group-B. . Blood transfusion requirement, either intra operatively or postoperatively was more in push method i.e Group-B. However the occurrence of atonic PPH was not significant in both groups.

Foetal distress was observed in 10 out of 100 cases out of which 4(7.27\%) cases observed in Group-A and $6(13.33 \%)$ cases in Group-B. Meconium stained liquor was observed in $10(18.18 \%)$ cases in Group-A and $12(26.6 \%)$ cases with meconium stained liquor was observed in Group-B. Still births were 4(7.27\%) cases observed in Group-A and 5(11.11\%) cases in Group-B. Birth asphyxia and still birth rates were almost similar in two groups, indicating that, the technique of delivery was not responsible for. The parameters of foetal outcome such as meconium stained liquor, birth asphyxia and still birth rates, were not related to the technique of foetal extraction but these were related to the outcome or complication of obstructed labour.(19,20) .

\section{Conclusions}

While complications are inherent in both methods, Patwardhan method of delivery of the fetus as well as reverse breech extraction for second stage labour has been shown to confer considerable advantage in prevention of maternal morbidity over the push method in our institution. Our findings support the fact that the Patwardhan method as well as reverse breech extraction could be a useful maneuver in intraoperative disengagement of fetal head, when encountered at second stage CS and it is our opinion that these manoeuvre can be practiced selectively as a primary technique.

\section{References}

[1]. AbouZahr C. Prolonged and obstructed labour. In: Murray CJL and Lopez AD, eds. Health dimensions of sex and reproduction: the global burden of sexually transmitted diseases, maternal conditions, perinatal disorders, and congenital anomalies. WHO, 1998.Chhabra S, Gandhi D, Jaiswal M. Obstructed labour - a preventable entity. J Obstet Gynaecol. 2000;20:151-53.

[2]. Murphy DJ, Liebling RE, Verity L, Swingler R, Patel R. Early maternal and neonatal morbidity associated with operative delivery in second stage of labour: a cohort study. Lancet. 2001;358:1203-7.

[3]. Hager RM, Daltviet AK, Hofoss D, Nilson ST, Oian P, Henriksen T. Complications of cesarean deliveries: rates and risk factors. Am J Obstet Gynecol. 2004;190:428-34.

[4]. Singh M, Varma R. Reducing complications associated with a deeply engaged head at caesarean section: A simple instrument. The Obstetrician \& Gynaecologist. 2008;10:38-41. 
[5]. Sung JF, Daniels KI, Brodzinsky L, El-Sayed YY, Caughey AB, Lyell DJ. Caesarean delivery outcome after a prolonged second stage of labor. Am J of Obstet Gynecol. 2007;197:306:1-

[6]. Alexander JM, Leveno KJ, Rouse DJ, Landon MB, Gilbert S, Spong CY. Comparison of maternal and fetal outcome from primary caesarean delivery during the second compared with the first stage of labor. Obstet Gynecol. 2007;109:917-21.

[7]. Lippert TH. Abdominovaginal delivery in case of impacted head in cesarean section operation. Am J Obstet Gynecol. 1985;151(5):703.

[8]. Landesman R, Graber EA. Abdomino-vaginal delivery: modification of the cesarean section operation to facilitate delivery of the impacted head. Am J Obstet Gynecol. 1984;148(6):707.

[9]. Fong YF, Arulkumaran S. Breech Extraction - an alternative method of delivering a deeply engaged head at cesarean section. International Journal of Obstetrics and Gynaecology. 1997;56:184-4.

[10]. Robert, L.B. (2012) Difficult Fetal Extraction at Cesarean Delivery: What Should You Do? Next Time You Face This Hazardous Scenario, Try Reverse Breech Extraction, 24, 1.

[11]. Patwardhan BD, Motashaw ND. Caesarean Section. J Obstet Gynecol India. 1957;8:1-15.

[12]. Murray Utah. Clinical Innovations. C-snorkel: Impacted Fetal Head Release Device by Softlift. 2012. Available at [http://www.clinicalinnovations.com/portfolioitems/c-snorke]. Accessed on 20th sep 2015.

[13]. Yifru Berhan, Asres Berhan. A metaanlysis of reverse breech extraction to deliver a deeply impacted head during cesarean delivery. International journal of gynecology and obstetrics. 2014;124:99-105.

[14]. Schwake, Petchenkin, Younis. Reverse breech extraction in cases of second stage caesarean section. Journal of Obstetrics and Gynaecology. 2012;32:548-51.

[15]. Blickstein I. Difficult delivery of the impacted fetal head during caesarean section: intraoperative disengagement dystocia. J Perinat Med. 2004;32:465-9.

[16]. Kafali H. Cesarean breech extraction for impacted fetal head in deep pelvis after a prolonged obstructed labour: a cesarean technique variation. Internet J Gynaeco. 2003;2-5.

[17]. Fasubaa OB, Ezechi OC, Orji EO, Ogunniyi SO, Akindlele ST, Loto OM et al. Delivery of the impacted head of the fetus at caesarean section after prolonged obstructed labour: A randomised comparative study of the two methods. J Obstet Gynaecol. 2002;22:375-8.

[18]. Chopra S, Bagga R, Keepanasseril A, Jain V, Kalra J, Suri V. Disengagement of the deeply engaged fetal head during cesarean section in advanced labor: conventional method versus reverse breech extraction. Acta Obstet Gynecol Scand. 2009;88:1 163-6.

[19]. Loudon JAZ, Groom KM, Hinkson L, Harrington D, Paterson-Brown S. Changing trends in operative delivery performed at full dilatation over a 10-year period. J Obstet Gynaecol. 2010;30:370-5.

[20]. Rhadha P, Tagore S, Rahman MFA, Tee J. Maternal and perinatal morbidity after caesarean delivery at full cervical dilatation. Singapore Med J. 2012;53:655-8. 